\title{
MODO DE VIDA RIBEIRINHO E A LONGITUDINALIDADE DO CUIDADO NA ATENÇÃO PRIMÁRIA EM SAÚDE
}

\section{WAY OF LIFE AND THE RIVERSIDE LONGITUDINALITY CARE IN PRIMARY HEALTH CARE}

\author{
Gisele de Brito Brasil', Diene Keli Assunção dos Santos', \\ Laura Maria Vidal Nogueira², Ivaneide Leal Ataíde Rodrigues ${ }^{2}$
}

\section{RESUMO}

Objetivos: identificar atitudes que são valorizadas pelos profissionais das equipes da Estratégia Saúde da Família Ribeirinhas relacionadas à longitudinalidade do cuidado; e verificar como ocorre o processo de vínculo longitudinal. Estudo com abordagem quantitativa realizado com quatro equipes da Estratégia Saúde da Família de Populações Ribeirinhas da região metropolitana de Belém/PA. Os dados foram coletados por meio de uma escala Likert e analisados de forma descritiva. Os participantes concordaram $(71 \%)$ que a equipe deve assistir a população de sua área adscrita de forma longitudinal, que o vínculo é importante para esse fortalecimento (94\%) e a falta de condições de trabalho pode comprometer esse cuidado (81\%). Embora o modelo Saúde Família favoreça a constituição de vínculo entre usuários e profissionais, as realidades de vida e de trabalho nos territórios ribeirinhos devem ser valorizadas para o cuidado longitudinal.

Descritores: Atenção Primária à Saúde; Saúde da Família; Continuidade daAssistência ao Paciente.
${ }^{1}$ Enfermeira Especialista em Saúde da Família pela Universidade do Estado do Pará (UEPA), Belém PA, Brasil.

${ }^{2}$ Doutora em Enfermagem pela Universidade Federal do Rio de Janeiro, UFRJ, Rio de Janeiro, RJ, Brasil.

\begin{abstract}
Objectives: To identify attitudes that are valued by professionals in the Health Strategy teams Riparian family-related care longitudinality; and check how is the longitudinal bonding process. Study with a quantitative approach carried out with four teams of the Health Strategy Riparian Populations of family metropolitan area of Belém / PA. Data were collected through a Likert scale and analyzed descriptively. Participants agreed (71\%) that the team should assist the population of its ascribed area longitudinally, that the link is important for this strengthening (94\%) and lack of working conditions can compromise this care (81\%). Although the Family Health model favors the formation of bond between users and professionals, the realities of living and working in the riparian areas should be valued for longitudinal care.
\end{abstract}

Descriptors: Primary Health Care; Family Health; Continuity of Patient Care 


\section{Introdução}

Um importante desafio para o Sistema Único de Saúde (SUS) na contemporaneidade é o alcance das populações mais vulneráveis, dentre elas, as comunidades ribeirinhas, muito expressivas na região amazônica, de modo especial no Estado do Pará. Nesse sentido estabeleceram-se estratégias para redução das desigualdades no acesso aos serviços de saúde com políticas de equidade, na perspectiva de atender as demandas de saúde desses povos.

Assim, o fortalecimento da Atenção Primária em Saúde (APS) se constitui uma possibilidade de inclusão dessas pessoas na assistência prestada pelo SUS, tendo em vista que é por meio da Estratégia Saúde da Família (ESF) que deve ocorrer o primeiro contato do indivíduo com o sistema de saúde. Assim a APS representa a porta de entrada da rede de serviços para atender suas necessidades e problemas de saúde.

A APS apresenta em sua constituição quatro atributos básicos essenciais para seu funcionamento, sendo eles: acessibilidade, integralidade, coordenação do cuidado e longitudinalidade. O termo longitudinalidade no contexto da APS representa uma relação pessoal de longa duração entre os usuários e os profissionais de saúde onde se estabelece um vínculo, uma relação terapêutica duradora entre os envolvidos ${ }^{1}$.

O lócus de ação da ESF é um território geograficamente definido, cujas atividades da equipe oportunizam a relação de longa duração com os usuários, independente das necessidades de saúde, adquirindo um sentido de "vínculo longitudinal"2.

Os moradores das comunidades ribeirinhas, apesar de muitas vezes se encontrarem geograficamente próximas aos grandes centros, têm dificuldades para acesso a assistência à saúde pela característica de residirem às margens de rios, igarapés e furos, em habitações distantes uma das outras, sem acesso facilitado aos centros urbanos ${ }^{3}$.

Partindo destas questões que permeiam o modo de vida das comunidades ribeirinhas, é possível identificar desafios peculiares para as equipes de saúde que as assistem, relacionados à baixa densidade demográfica, tendo em vista a dispersão dos moradores na floresta ao longo do curso dos rios. Esse cenário, presente no território, remete à dificuldades operacionais para o trabalho, pela indisponibilidade de transporte para oportuno deslocamento da equipe, repercutindo na baixa frequência de contato da equipe de saúde com os moradores. Dessa forma é possível visualizar influência significativa na ocorrência da longitudinalidade do cuidado com esta população dificultando a atenção à saúde de qualidade e respeitando suas particularidades 4 .

Assim torna-se fundamental saber como se desenvolve esse cuidado de forma longitudinal pela Equipe de Saúde da Família em uma comunidade ribeirinha, partindo da premissa de que o vínculo criado por esta longitudinalidade é fator importante para a qualidade da saúde, e tende a melhorar o conhecimento dos reais problemas da população atendida pelos serviços, além de facilitar o relacionamento dos usuários com os profissionais que os atendem ${ }^{5}$.

Dessa forma, definiram-se como objetivos desta pesquisa: Identificar as atitudes que são valorizadas pelos profissionais das equipes de Estratégia Saúde da Família Ribeirinhas em relação a longitudinalidade do cuidado no território; verificar como ocorre o processo de vínculo longitudinal entre os profissionais e usuários.

\section{Metodologia}

Trata-se de um estudo com abordagem quantitativa realizado com quatro equipes da ESF que atendem população ribeirinha na região metropolitana de Belém/PA, sendo duas localizadas na capital paraense (USF Combú, USF Furo das Marinhas) e duas no município de Benevides (USF Paraíso do Murinin). Essas quatro equipes representam $66,6 \%$ do total de equipes que desenvolvem suas atividades junto aos ribeirinhos da região.

A população do estudo foi de 32 profissionais que integram as quatro equipes e a amostra constituída por 21 profissionais, que representa $65,6 \%$. Todas as categorias profissionais existentes nas equipes integraram o estudo, a saber: agentes comunitários de saúde, enfermeiros, médicos, odontólogo e técnicos de enfermagem. Definiu-se como critério de inclusão: integrar a equipe há pelo menos seis meses e encontrar-se em atividade por ocasião da coleta de dados.

Para obtenção dos dados utilizou-se um instrumento composto de duas partes: a primeira referente à caracterização sociodemográfica dos profissionais; e a segunda correspondente a uma escala Likert adaptada pelos pesquisadores para medir o grau de acordo ou desacordo em cada uma das proposições apresentadas a respeito da longitudinalidade do cuidado. Para tanto os participantes eram solicitados a assinalar o grau de acordo ou desacordo em cada proposição, tendo como opções "discordo totalmente", "discordo", "discordo em parte", "concordo", "concordo totalmente". 
A coleta dos dados se efetivou nas dependências das Unidades Saúde da Família de forma individualizada e privativa. As respostas a respeito do nível concordância foram agrupadas em três grandes eixos (Quadro 1) para facilitar o entendimento e análise dos mesmos, são eles: longitudinalidade do cuidado, vínculo e estrutura. E no tratamento dos dados, as afirmativas "discordo" e "concordo em parte" foram agrupadas por apresentarem o mesmo valor de resposta.

Quadro 1 - Eixos de análise

\begin{tabular}{|c|c|}
\hline Eixos & Proposições \\
\hline \multirow{5}{*}{ Longitudinalidade do cuidado } & $\begin{array}{c}1 \text { - O vínculo entre equipe de saúde-usuário é essencial } \\
\text { à longitudinalidade. }\end{array}$ \\
\hline & $\begin{array}{l}2 \text { - Na equipe de saúde todos tem em suas atribuições } \\
\text { básicas a prestação de uma assistência longitudinal. }\end{array}$ \\
\hline & $\begin{array}{c}3 \text { - O cuidado longitudinal somente deve ocorrer } \\
\text { aos que tem uma maior necessidade deste como os } \\
\text { doentes crônicos. }\end{array}$ \\
\hline & $\begin{array}{l}4 \text { - Longitudinalidade do cuidado requer atendimento } \\
\text { prioritário à grupos mais vulneráveis. }\end{array}$ \\
\hline & $\begin{array}{l}5 \text { - Longitudinalidade do cuidado implica no } \\
\text { acompanhamento do usuário em todos os seus ciclos } \\
\text { de vida. }\end{array}$ \\
\hline \multirow{4}{*}{ Vínculo } & $\begin{array}{c}6 \text { - Para ter vínculo com a pessoa, tem que saber } \\
\text { ouvi-lás. }\end{array}$ \\
\hline & $\begin{array}{c}7 \text { - Vínculo é estabelecer uma relação de amizade e } \\
\text { companheirismo, não só aquela relação de paciente e } \\
\text { profissional. }\end{array}$ \\
\hline & $\begin{array}{l}8 \text { - A visita domiciliar é uma forma de vinculo e assim } \\
\text { de longitudinalidade. }\end{array}$ \\
\hline & $\begin{array}{l}9 \text { - O vínculo é necessário ao desenvolvimento das } \\
\text { ações de promoção, prevenção, controle, cura e } \\
\text { recuperação. }\end{array}$ \\
\hline Estrutura & $\begin{array}{c}10 \text { - A falta de condições de trabalho como estrutura da } \\
\text { unidade, falta de transporte interferem na criação de } \\
\text { vínculo e consequentemente na longitudinalidade do } \\
\text { cuidado aos usuários. }\end{array}$ \\
\hline
\end{tabular}

Os dados obtidos foram inseridos em planilhas eletrônicas no Microsoft Excel e posteriormente analisados de forma descritiva utilizando frequência absoluta e relativa com as seguintes variáveis: faixa etária; sexo; formação profissional; número de empregos atuais; vínculo empregatício; tempo de atuação na ESF; e escore de longitudinalidade do cuidado, vínculo e estrutura dos serviços. Posteriormente, foram construídos gráficos e tabelas de modo a expressar melhor 0 conteúdo. Os dados foram interpretados e discutidos com base na literatura disponível.

Em respeito às questões éticas, Resolução 466/2012 - CNS/MS, o estudo foi aprovado no Comitê de Ética em Pesquisa do Curso de Graduação em Enfermagem da Universidade do Estado do Pará, parecer n 840.085 na data de 21/10/2014, e a coleta de dados aconteceu mediante autorização das Secretarias Municipais de Saúde dos municípios de Belém e Benevides.

\section{Resultados}

Primeiramente serão apresentados os dados referentes à caracterização sociodemográfica das equipes, e em seguida, aqueles resultantes das respostas dos profissionais quanto ao trabalho junto à população ribeirinha. 
Ao analisar as características sociodemográficas, identificou-se predomínio do sexo feminino com 81\% (17) e faixa etária de 26-35 anos com 52\% (11). Quanto a área de especialização dos nove profissionais que possuíam nível superior completo, 33\% (3) possuíam especialidade em saúde da família, 22\% (2), possuíam especialidade em enfermagem do trabalho e 11\%(1), especialidade em UTI neonatal e adulto (Tabela 1).

No que se refere ao número de empregos atuais, $62 \%$ (13) dos profissionais referiram possuir apenas um vínculo e $24 \%$ (5) possuíam dois ou três empregos. Em relação ao tipo de vínculo institucional, $76 \%$ (16) referiram que trabalhavam mediante contrato estabelecido com a gestão municipal. Do total de participantes, 29\% (6) possuíam menos de um ano de atuação na ESF e 24\% (5) mais de cinco anos de trabalho na estratégia (Tabela 1).

Tabela 1 - Caracterização sociodemográfica dos profissionais da ESF ribeirinha da região metropolitana de Belém. Pará, 2014.

\begin{tabular}{|c|c|c|}
\hline Variáveis & $\mathbf{F a}$ & Fr\% \\
\hline \multicolumn{3}{|l|}{ Faixa etária } \\
\hline$<=25$ & 2 & 10 \\
\hline $26-35$ & 11 & 52 \\
\hline $36-45$ & 5 & 24 \\
\hline $46-55$ & 3 & 14 \\
\hline \multicolumn{3}{|l|}{ Sexo } \\
\hline Feminino & 17 & 81 \\
\hline Masculino & 4 & 19 \\
\hline \multicolumn{3}{|c|}{ Especialização-nível superior } \\
\hline Enfermagem do trabalho & 2 & 22 \\
\hline UTI neonatal e adulto & 1 & 11 \\
\hline Saúde da família & 3 & 33 \\
\hline Outros não informado & 3 & 33 \\
\hline \multicolumn{3}{|l|}{$\mathbf{N}^{\circ}$ de empregos atuais } \\
\hline 1 & 13 & 62 \\
\hline 2 & 4 & 19 \\
\hline 3 & 1 & 5 \\
\hline não informou & 3 & 14 \\
\hline \multicolumn{3}{|l|}{ Vínculo empregatício } \\
\hline Concurso público & 5 & 24 \\
\hline Contrato & 16 & 76 \\
\hline \multicolumn{3}{|l|}{ Tempo na ESF } \\
\hline menos de 1 ano & 6 & 29 \\
\hline entre 1 e 2 anos & 5 & 24 \\
\hline entre 3 e 4 anos & 3 & 14 \\
\hline entre 7 e 8 anos & 1 & 5 \\
\hline entre 9 e 10 anos & 1 & 5 \\
\hline maior que 10 anos & 5 & 24 \\
\hline
\end{tabular}


Dentre as proposições do eixo longitudinalidade do cuidado, podemos destacar a prioridade para o cuidado longitudinal referente aos grupos prioritários, em que 52\% (11), concordaram em parte com essa proposição (Figura 1).

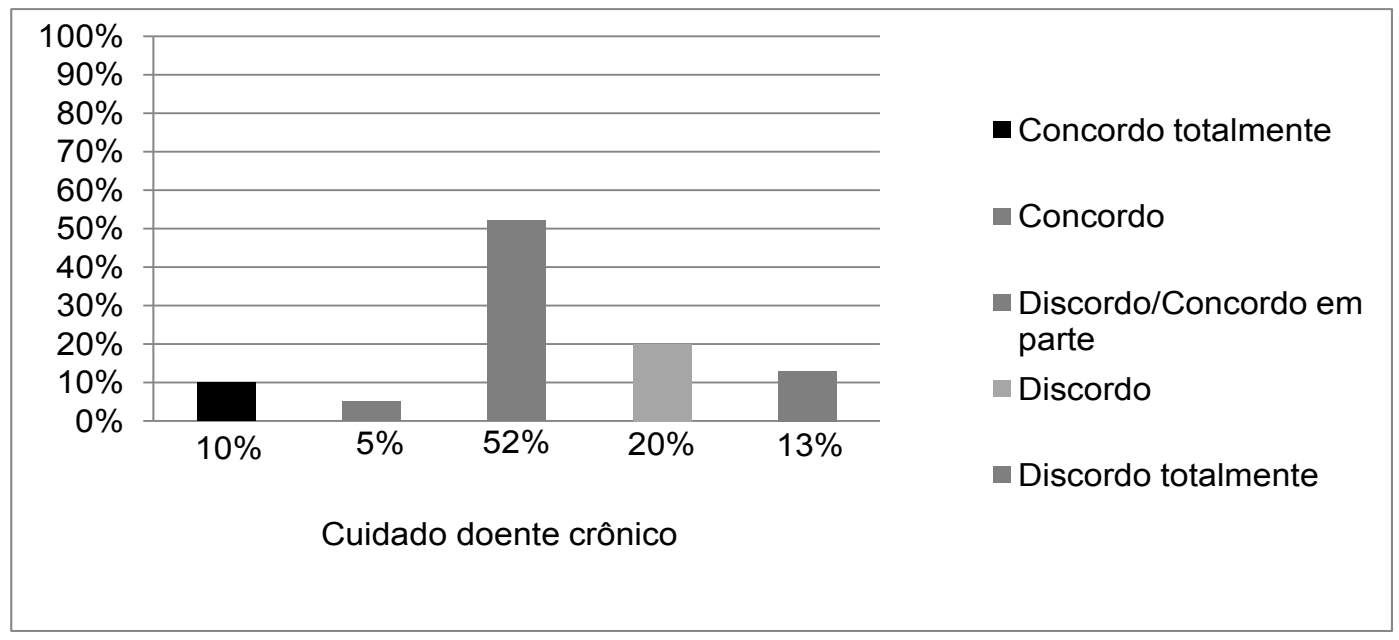

Figura 1. Concordância do cuidado longitudinal com aqueles que têm maior necessidade de atenção à saúde, como os doentes crônicos, dentre os profissionais da ESF ribeirinha da região metropolitana de Belém. Pará, 2014.

Merece destaque a concordância em $67 \%$ (14), para a priorização do atendimento aos grupos mais vulneráveis, correspondente a somatória de $48 \%$ (10) daqueles que "concordaram totalmente" com 19\% (4) que "concordaram". Nas demais proposições abordadas sobre o eixo longitudinalidade do cuidado, os resultados mais expressivos foram para 0 "relacionamento entre a equipe e usuário" com 76\% (16), e 43\% (9) que "concordaram toltamente" que a longitudinalidade se dá nas atribuições específicas da equipe para com o usuário ribeirinho. A esta proposição, se somado os 52\% (11) que "concordaram", visualiza-se expressividade nos resultados. Com relevância, da mesma forma, foi citado o acompanhamento nos diversos ciclos de vida, com 52\% (11) que, "concordam totalmente", com a proposição (Figura 2).

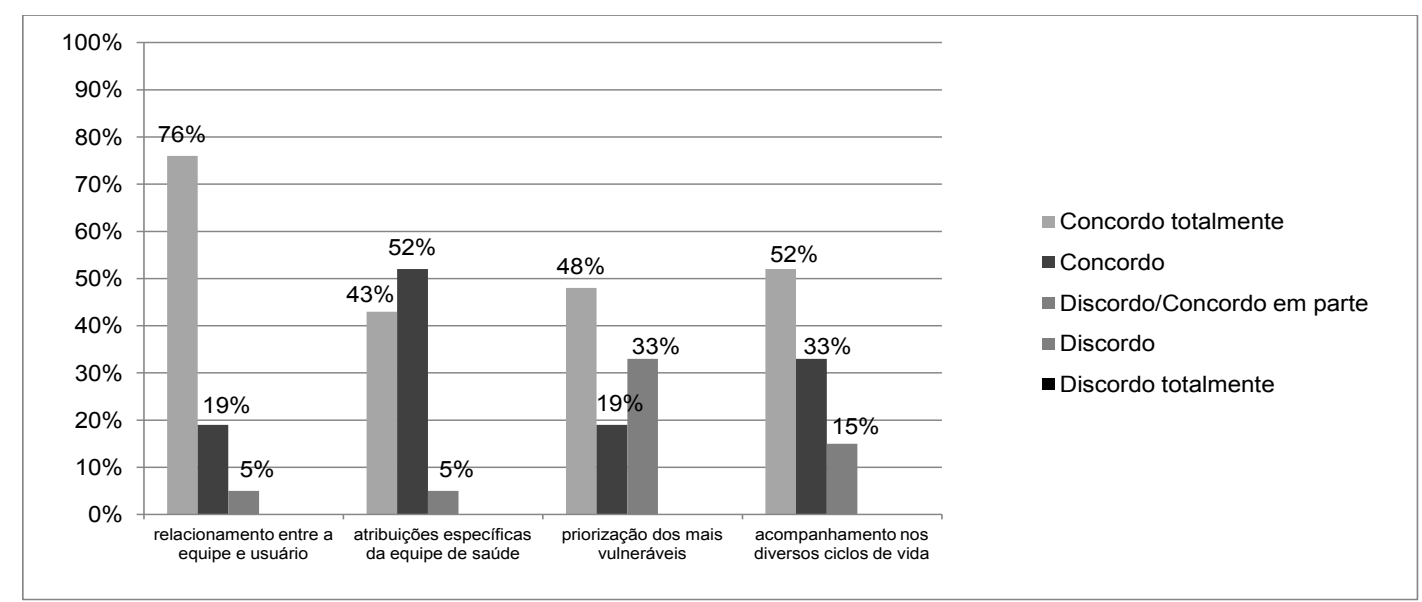

Figura 2. Fatores determinantes para a longitudinalidade do cuidado dentre os profissionais da ESF ribeirinha da região metropolitana de Belém. Pará, 2014

De acordo com o eixo vínculo, as proposições que favorecem a sua formação é a escuta atenta com 100\% (21) de concordância, decorrente da somatória "concordo totalmente" 76\% (16) e "concordo" 24\% (5). Da mesma forma, a visita domiciliar também foi considerada importante, com 95\% (20) de concordância, sendo 52\%(11) para "concordo totalmente" e 43\%(9) para "concordo" (Figura 3). 


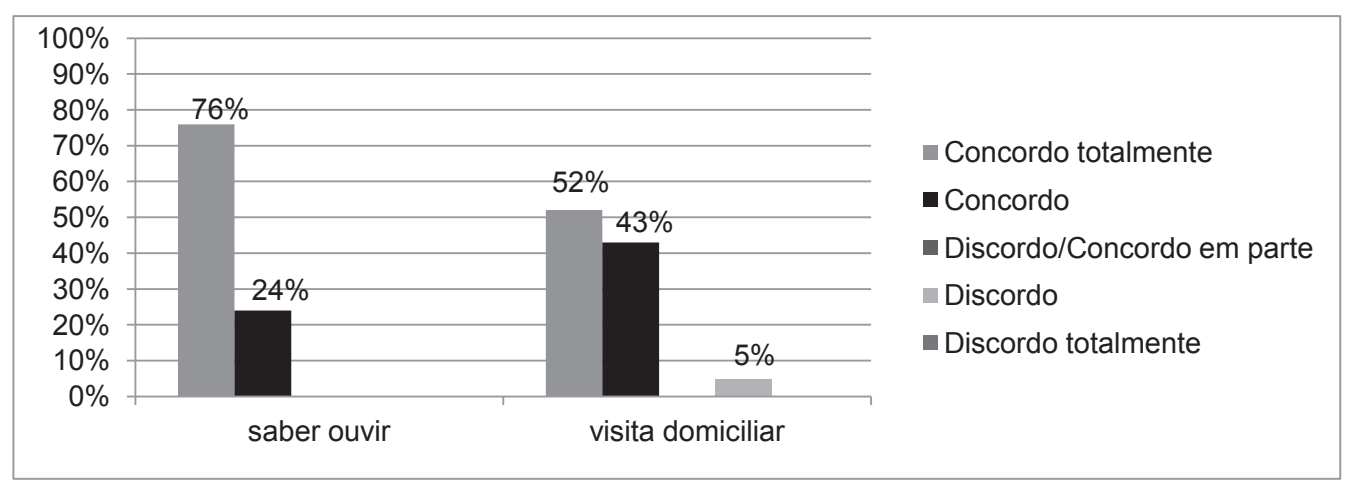

Figura 3. Atitudes/atividades que favorecem o vínculo dentre os profissionais da ESF ribeirinha da região metropolitana de Belém. Pará, 2014.

Referente ao eixo estrutura, $57 \%$ (12) e 14\% (3) "concordaram totalmente" e "concordaram" que a falta de condições de trabalho, a exemplo da estrutura deficitária na unidade de saúde e a falta de transporte para deslocamento da equipe e usuários impactam na criação de vínculo e assim na longitudinalidade (Figura 4).

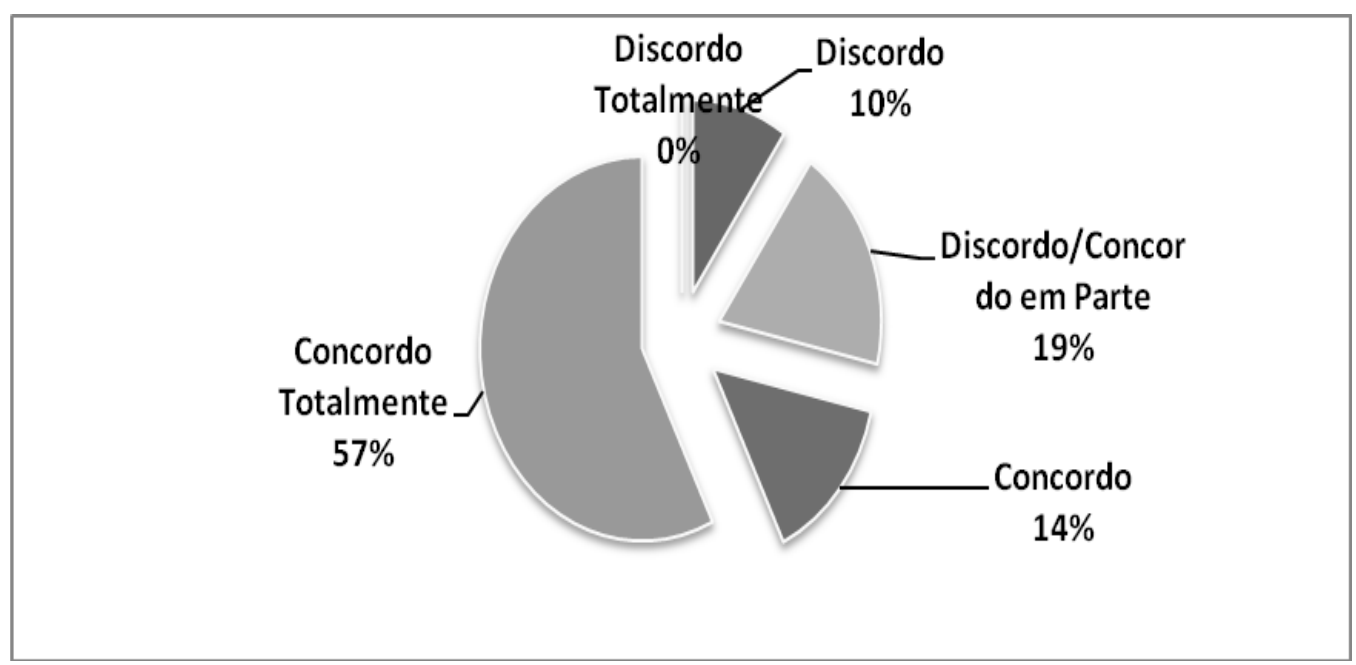

Figura 4. Concordância no eixo estrutura e falta de condições de trabalho como interferem na longitudinalidade do cuidado dentre os profissionais da ESF ribeirinha da região metropolitana de Belém. Pará, 2014.

\section{Discussão}

A realidade enfrentada pelos profissionais que atendem as comunidades ribeirinhas é peculiar, uma vez que além da necessidade de conhecer os dispositivos da APS, devem ter apropriação das questões culturais locais. Em razão das características do território, essas equipes enfrentam dificuldades de logística no dia-a-dia nas ilhas, principalmente aquelas relativas a transporte público coletivo e institucional. Faz-se necessária formação específica para atender as demandas próprias do modo de vida ribeirinho.

Os dados sociodemográficos nos revelaram que somente $33 \%$ (3) dos profissionais de nível superior possuíam especialidade em saúde da família. Nesse sentido, estudo realizado em 20 municípios do Rio Grande do Sul, acerca do perfil socioprofissional das equipes de ESF, concluiu que a qualificação dos profissionais é um aspecto frágil , apontando a necessidade de fortalecimento na formação, capaz de proporcionar mudanças assistenciais frente às demandas da ESF, valorizando o aprimoramento técnico-científico para as diferentes necessidades ${ }^{7}$. 
Merece destaque a existência de múltiplos vínculos empregatícios entre os profissionais das equipes de saúde, o que pode interferir nas práticas diárias desenvolvidas no território. Os profissionais, por muitas vezes, exercem atividades complementares que geram rendimentos melhores por mais que estejam vinculados à ESF ${ }^{8}$. De acordo com a regulamentação ministerial, os profissionais devem cumprir carga horária de quarenta horas semanais de trabalho, a exceção do médico ${ }^{9}$. Vale ressaltar que a existência de mais de um vínculo pode ser motivada pela preocupação de muitos profissionais com a instabilidade, haja vista que $76 \%$ (16) trabalhavam em regime de contrato, um vínculo empregatício precário, porém crescente dentre os trabalhadores do SUS.

O tempo de atuação na ESF é relevante para efetivação do vínculo e consequentemente para a longitudinalidade do cuidado, pois a grande rotatividade de profissionais produz a quebra nos processos de trabalho, demandando tempo e esforço da equipe quanto ao conhecimento do quadro de saúde local e dos valores culturais. Isso implica diretamente no elo de confiança da equipe de saúde com a população. Um estudo realizado no ano de 2013 sobre longitudinalidade concluiu que a permanência reduzida dos profissionais de saúde na ESF se constitui um dos fatores críticos para 0 sucesso da ESF no Brasil. O mesmo estudo apontou ainda que a remuneração atrai os profissionais para trabalhar na ESF, mas não os fixa, remetendo a outras questões como a preocupação com a aposentadoria, visto que o ganho salarial reduz pela perda da remuneração referente ao trabalho na ESF quando os profissionais não estão mais em atividade ${ }^{10}$.

No eixo longitudinalidade do cuidado, é importante destacar o entendimento dos profissionais em relação à priorização do atendimento aos que tem maior necessidade, como os doentes crônicos. Da mesma forma, foi evidenciado em estudo similar que os profissionais desenvolvem a longitudinalidade do cuidado, essencialmente, com pessoas nas fases da vida que exigem mais atenção e/ou que estão contemplados em programas com maior incentivo ou mesmo cobrança por parte das Secretarias de Saúde e gestores, dentre eles os que apresentam condição crônica ${ }^{11}$.

Neste estudo, no eixo vínculo, podemos destacar a priorização convergente a respeito da importância de saber ouvir, para formação de vínculo profissional. Essa atitude parte da premissa que o profissional deve ter uma escuta ativa e sensível exigindo uma relação de confiança mútua. O vínculo se dá com escuta, convivência e proximidade, valorizando o "saber ouvir", "ser igual" e "não atribuir diferenças"12. Na população ribeirinha, a escuta deve levar em consideração as características locais que configuram o modo de vida e as peculiaridades da população.

Outro aspecto favorecedor do vínculo é a realização de visitas domiciliares de forma regular e sistemática. Esta é uma atividade comum a todos os membros da equipe de saúde da família, exigindo dos profissionais o conhecimento da realidade local, além do trabalho em equipe. Um estudo realizado com agentes comunitários de saúde destacou que ao visitarem diariamente as famílias de sua área, adquirem cada vez mais conhecimento sobre a realidade da população. Por isso, quando adequadamente capacitados, tem condições de elencar as prioridades e levar as diversas situações de saúde para os demais membros da equipe, para que juntos possam discutir e planejar a melhor solução dos casos ${ }^{13}$.

Estudo realizado no Rio Grande do Sul concluiu que um dos principais problemas no trabalho da ESF é a falta de infraestrutura nas Unidades de Saúde e ausência de transporte para as visitas domiciliares como um fator que inviabiliza a adequada realização das atividades diárias, principalmente nas localidades mais afastadas ou de difícil acesso. Também a escassez de materiais e equipamentos, medicamentos e insumos, levam a não oferta de determinadas ações. Enfrentam ainda, dificuldades como a falta de apoio dos órgãos responsáveis pela ESF, recursos humanos, vontade política, repasse de verbas para as prefeituras e/ou aplicação indevida das mesmas ${ }^{14}$.

Assim, a longitudinalidade do cuidado requer uma prática profissional com importante grau de comunicação, interpretação, negociação e responsabilidades compartilhadas, estimulando o vínculo e ampliando o acesso das populações ribeirinhas e as percepções dos profissionais acerca das suas singularidades culturais e necessidades de cuidado ${ }^{12}$.

\section{Considerações Finais}

As atitudes que são valorizadas pelos profissionais das equipes de Estratégia Saúde da Família, da região metropolitana de Belém, que atendem comunidades ribeirinhas, em relação à longitudinalidade do cuidado estão permeadas pelo estabelecimento do vínculo dos profissionais com o usuário/família/comunidade, atendimento prioritário a grupos mais vulneráveis e fatores como qualificação profissional, tipo de vínculo empregatício e tempo de atuação na ESF.

O estabelecimento de vínculo longitudinal está intimamente relacionado a atitude de saber ouvir por parte da equipe e, pela realização de visita domiciliar. Há de se ressaltar que a falta de condições de trabalho como estrutura da Unidade e falta de transporte interferem na criação de vínculo e consequentemente na longitudinalidade do cuidado. 
Desse modo, o conhecimento produzido pela pesquisa permite identificar a importância do preparo da equipe local de saúde e a efetivação do vínculo longitudinal para à atenção integral nos territórios, contribuindo com a efetivação dos princípios da APS.

\section{Referências}

1. Starfield B. Atenção Primária: equilíbrio entre necessidades de saúde, serviços e tecnologia. Brasília: UNESCO, Ministério da Saúde; 2002.

2. Baratieri T, Marcon SS. Longitudinalidade do cuidado: Compreensão dos enfermeiros que atuam na Estratégia Saúde da Família. Esc Anna Nery. 2011 out-dez; 15(4): 802-10.

3. Ferreira LSC, Costa JHG, Costa CA, Melo MFC, Andrande ML, Martins LC et al. Soroprevalência do vírus linfotrópico de células T humanas. Rev Pan-Amaz Saude. 2010;1(3):103-108.

4. Brasil GB, Matos ASB. Práticas de cuidados em saúde com crianças ribeirinhas de 0 a 2 anos da llha do Combú, Belém/ PA. Trabalho de Conclusão de Curso (Graduação em Enfermagem) - Universidade do Estado do Pará, Belém do Pará. 2012. 5. Brunello MEF, Ponce MAS, Assis EG, Andrade RLP, Scatena LM, Palha PF et al. O vínculo na atenção à saúde: revisão sistematizada na literatura, Brasil (1998-2007). Acta Paul Enferm. 2010; 23(1):131-5.

6. Conselho Nacional de Saúde. Resolução n 466 de 12 dezembro de 2012. [Internet]. Disponível em http://conselho. saude.gov.br/resolucoes/2012/Res0466.pdf Acesso em: 10 nov 2013.

7. Zanetti TG, Sand ICPV, Perlini NMOG, Kopf AW, Abreu PB. Perfil socioprofissional e formação de profissionais de equipes de saúde da família: um estudo de caso. Cienc Cuid Saude. 2010 jul-set; 9(3):448-55.

8. Medeiros CRG, Junqueira AGW, Schwinge G, Carreno I, Jungles LAP, Saldanha OMFL. A rotatividade de enfermeiros e médicos: um impasse na implementação da Estratégia de Saúde da Família. Ciência \& Saúde Coletiva. 2010; 15 (Supl. 1): 1521-31.

9. Brasil MS. Política Nacional de Atenção Básica. Brasília, 2012.

10. Giovani MSP, Vieira CM. Longitudinalidade do cuidado diante da rotatividade de profissionais na Estratégia Saúde da Família. RECIIS - Rev. Eletr. de Com. Inf. Inov. Saúde. [Internet]. 2013 dez; 7 (4). Disponível em: www.reciis.icict.fiocruz.br. 11. Baratieri T, Mandú ENT, Marcon SSM. Compreensão de enfermeiros sobre vínculo e longitudinalidade do cuidado na estratégia saúde da família. Ciencia y Enfermerla XVIII (2): 11-22, 2012.

12. Baratieri T. Percepções de enfermeiros sobre a longitudinalidade em seu trabalho na Estratégia Saúde da Família. Dissertação (Mestrado em Enfermagem). Universidade Estadual de Maringá; 2010.

13. Sakata NM, Mishima SM. Articulação das ações e interação dos Agentes Comunitários de Saúde na equipe de Saúde da Família. Rev Esc Enfermagem USP 2012; 46(3):665-72.

14. Marqui ABT, Jahn AC, Resta DG, Colomé ICS, Rosa N, Zanon T. Caracterização das equipes da Saúde da família e de seu processo de trabalho. Rev Esc Enfermagem USP 2010; 44 (4): 956-61.

\section{Gisele de Brito Brasil}

Endereço para correspondência - Rua: Travessa G, Conj. Cohab Gleba 2, n¹52,

Bairro: Marambaia , CEP: 66623-316, Belém, PA, Brasil.

E-mail: gibrasilis@hotmail.com

Lattes: http://lattes.cnpq.br/3219750418090106

Diene Keli Assunção dos Santos - kelibelem2@yahoo.com.br

Laura Maria Vidal Nogueira - lauramavidal@gmail.com

Ivaneide Leal Ataíde Rodrigues - ilar@globo.com

\section{Enviado em 03 de setembro de 2015. Aceito em 13 de janeiro de 2016.}

